\title{
EL PERFIL LABORAL DE LOS LICENCIADOS EN CIENCIAS DE LA ACTIVIDAD FÍSICA Y DEL DEPORTE EN LA COMUNIDAD VALENCIANA, ESPAÑ
}

Antonio Campos Izquierdo

María Dolores González Rivera

\section{Resumen}

En este artículo se detallan las funciones laborales de actividad física y deporte desarrolladas por los Licenciados en Ciencias de la Actividad Física y del Deporte y sus características laborales en la Comunidad Valenciana (España). La metodología utilizada es cuantitativa de corte descriptivo, donde los procedimientos que se han seguido son los propios de la encuesta. Se ha realizado entrevistas individuales, orales y estandarizadas por medio de cuestionario a 600 personas que trabajaban en funciones de actividad física y deporte. En el estudio se obtiene que las principales funciones de los Licenciados en Ciencias de la Actividad Física y del Deporte son la docencia de la asignatura de Educación Física, la dirección y organización de actividad física y deporte, el entrenamiento deportivo y el cuidado y mantenimiento de la forma física para grupos. Además, se obtiene una elevada plurifuncionalidad, pluriempleo, movilidad laboral, contratación temporal y jornada laboral mayor de 40 horas, lo que conforma cierta precariedad e inestabilidad laboral, aunque es inferior a la de otras titulaciones. Las relaciones laborales sin contrato son muy significativas y los Licenciados trabajan para diversas entidades, pero son mayoría los que lo hacen para entidades públicas.

\section{Palabras-Clave}

Licenciado; Profesional; Deporte; Educación Física; Empleo.

\section{THE LABOUR PROFILE OF THE GRADUATES IN SCIENCES OF THE PHYSICAL ACTIVITY AND OF THE SPORT IN COMUNIDAD VALENCIANA, SPAIN}

Antonio Campos Izquierdo

María Dolores González Rivera

\begin{abstract}
In this article there are detailed the labour functions of physical activity and sport developed by the graduates in Sciences of the Physical Activity and of the Sport and his labour characteristics in the Comunidad Valenciana (Spain). The used methodology is quantitative of descriptive cut, where the procedures that have followed sound the own ones of the survey. There were realized individual, oral interviews standardized by means of questionnaire to 600 persons who were employed at functions of physical activity and deport. In the study there is obtained that the graduates in Sciences of the Physical Activity and of the Sport the principal functions that they realize are the teaching of the subject of Physical Education, the direction and organization of physical activity and sport, the sports training and the care and maintenance of the physical form for groups. Besides, a high plurifuncionalidad is obtained, pluriempleo, labour mobility, temporary contracting and labour major day of 40 hours, which shapes certain precariousness and labour instability, though it is lower than that of other qualifications. The
\end{abstract}


labour relations without contract are very significant and the majority of the graduates work for diverse organizations, but principally they do it for public organizations.

\section{Key-Words}

Graduates; Professional; Sport; Physical Education; Employment. 


\section{INTRODUCCIÓN}

La actividad física y el deporte es un factor generador de empleo, aunque es difícil de cuantificar y de delimitar (MADELLA, 2003; ALMEIDA, 2007), en Europa representan alrededor del 2\% de los puestos de trabajo (CAMY; CHANTELAT; LE ROUX, 1999). Por su parte, en España se estima en 200.000 los empleos directos sobre actividad física y deporte en España, a lo que habría que sumarle los empleos indirectos (CONSEJO SUPERIOR DE DEPORTES, 2000).

Además, en las últimas décadas, la tendencia del empleo en las funciones laborales de actividad física y deporte en España ha sido de crecimiento y diversificación, en el que también hay una tendencia de crecimiento de las funciones laborales no predominantes en décadas anteriores (CAMERINO; MIRANDA; PIGEASSOÚ, 1995; MARTÍNEZ DEL CASTILLO; PUIG, 2002; CAMPOS IZQUIERDO, 2008).

Asimismo, el anteproyecto de Ley de Ordenación del Ejercicio de las Profesiones del Deporte (CONSEJO SUPERIOR DE DEPORTES, 2009), determina que para trabajar en funciones de actividad física y deporte se debe poseer la titulación de actividad física y deporte correspondiente para cada función laboral. En la actualidad, en España las titulaciones oficiales de actividad física y deporte son las expuestas a continuación en la Tabla 1.

Tabla 1-Titulaciones de Actividad Física y Deporte en España

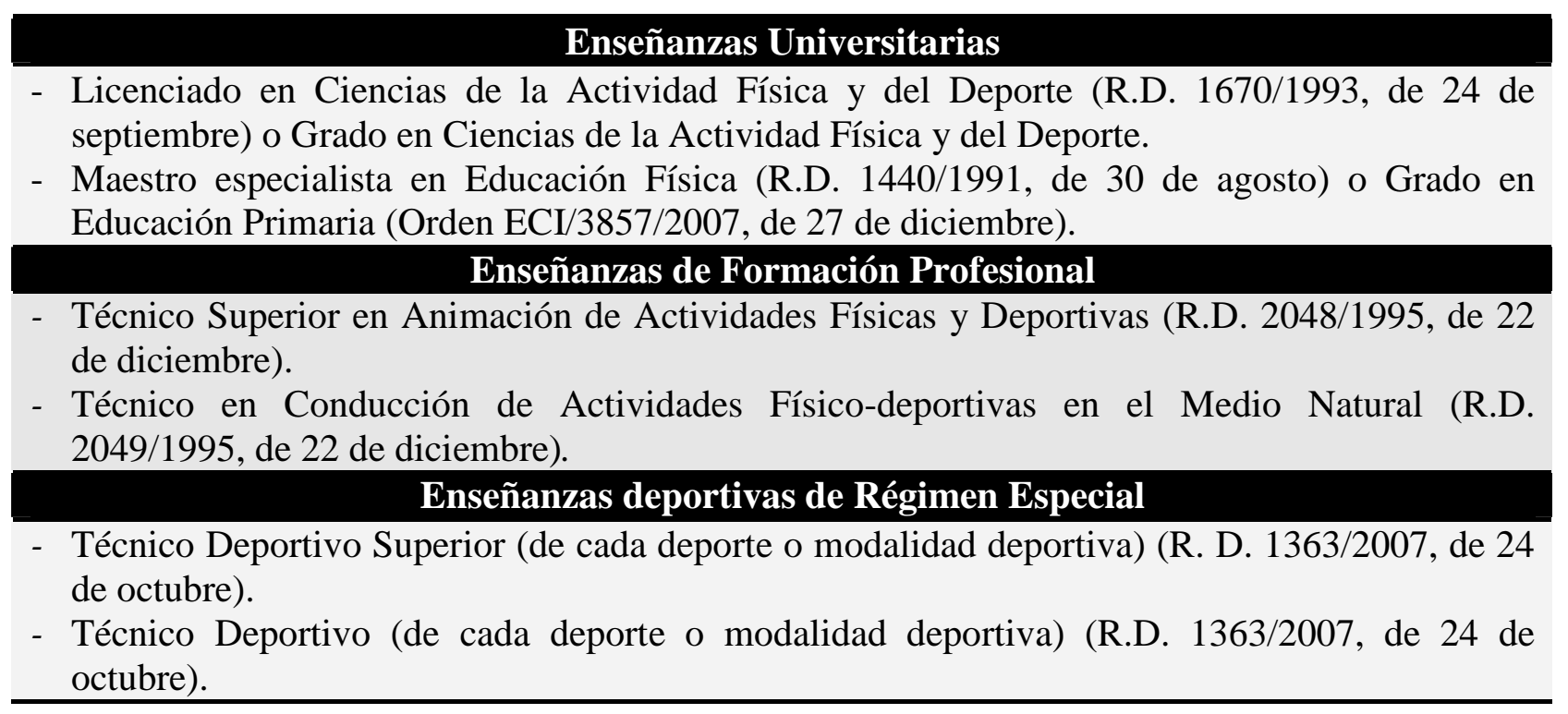

Conexões: revista da Faculdade de Educação Física da UNICAMP, Campinas, v. 8, n. 2, p. 01-18, maio/ago. 2010. 
No obstante, hay que remarcar que existe un gran porcentaje de personas que están trabajando en funciones de actividad física y deporte que no tiene ninguno de los diferentes tipos de titulaciones de la actividad física y del deporte expuestas en la Tabla 1 (MCMURTIE, 1997; CAMY; CHANTELAT; LE ROUX, 1999; AUGUSTIN, 2003). Además, hay que sumarle a este porcentaje de personas no tituladas, que muchas de las personas que tienen alguna de las distintas titulaciones de actividad física y deporte realizan funciones para las que su formación en la titulación que poseen no les capacita (por ejemplo: un técnico deportivo de fútbol que enseña natación). Todo ello muestra que es escaso el porcentaje (alrededor del 30\%) de las personas que trabajan en funciones de actividad física y deporte que tienen titulaciones de la actividad física y del deporte y realizan funciones para las que son competentes (CAMPOS IZQUIERDO, 2007a).

Hay que recodar que la actividad física y el deporte tiene un interés social y público importante, debido a que genera beneficios en las personas respecto a su salud, educación, aspectos éticos y sociales, así como los económicos y laborales en la sociedad, que son evidentes y demostrados en múltiples estudios e investigaciones. Pero si la actividad física y el deporte no es dirigida, diseñada, planificada, desarrollada, organizada o evaluada por una persona con la titulación de actividad física y deporte correspondiente, estos beneficios se pueden convertir en perjuicios y riesgos para la salud, la seguridad y la educación de los ciudadanos y para la sociedad; además de determinar la calidad y eficiencia de los servicios de actividad física y deporte (CAMPOS IZQUIERDO, 2007b). Igualmente, como explica Jiménez (2001), habrá que tener en cuenta la responsabilidad civil y penal de las personas que trabajan en funciones de actividad física y deporte sin la titulación adecuada y las entidades que los selecciona para desarrollar dichas funciones.

Dentro de estos titulados, como se ha descrito en la Tabla 1, están los licenciados en Ciencias de la Actividad Física y del Deporte. Estos titulados se forman en la universidad en la licenciatura en Ciencias de la Actividad Física y del Deporte, en la cual, en la actualidad, se está produciendo el proceso de la Convergencia Europea. Todo esto se debe a los procesos de cambio y transformación de la sociedad española y europea y del mercado laboral, donde las instituciones realizan el esfuerzo de ajustar las demandas de los ciudadanos y profesionales a la sociedad actual. En este proceso, la licenciatura se va a denominar grado en Ciencias de la Actividad Física y del Deporte. Esta titulación universitaria sobre la actividad física y el deporte tiene diversos objetivos y salidas profesiones (CASTELLANI FILHO, 2002; TAKS et al., 2003; TOJAL, 2004; VERENGUER, 2005; DANTAS, 2008; PETRY; FROBERG; 
MADELLA, 2008). En España, según la Conferencia de Decanos de Facultades de Ciencias de la Actividad Física y del Deporte (2004) los objetivos y las funciones de estos estudios deberán ser:

- La formación inicial de docentes de Educación Física en el sistema educativo;

- La formación de profesionales especialistas en la actividad física para la salud.;

- La formación de especialistas en entrenamiento deportivo, en la iniciación y en el alto rendimiento deportivo;

- La preparación de profesionales expertos en planificación, organización y dirección de actividad física y deporte y ocio, tanto en instituciones públicas como privadas.

Respecto al perfil laboral del licenciado en Ciencias de la Actividad Física y del Deporte, Martínez del Castillo (1991) determina que el 55\% trabajaban en docencia en la asignatura de Educación Física, el $18 \%$ en entrenamiento, el $14 \%$ en mantenimiento físico, el $11 \%$ como director y el $2 \%$ en animación deportiva. Asimismo, observó que según avanzaba la edad, estos titulados tendían a empleos de mayor responsabilidad y mejores condiciones laborales como son los de profesor de Educación Física y director de actividad física y deporte. El 78\% trabajaban para entidades públicas, el 11\% para empresas y el 7,8\% para asociaciones, donde el 3,2\% eran autónomos. Asimismo, el 73,5\% tenía contrato indefinido y la dedicación a tiempo completo era del $64,1 \%$, el $26,5 \%$ trabajaba menos de 20 horas, el $58 \%$ entre 20 y 40 horas y más de 40 horas el 15,5\%.

Por su parte, Rivadeneyra (1998) y Puig; Viñas (2006) obtienen que el mayor ámbito de intervención profesional de los Licenciados en Ciencias de la Actividad Física y del Deporte es ser profesor de Educación Física, así como que poseen una elevada polifuncionalidad, pluriempleo y contratación temporal.

El estudio de la Conferencia de Decanos de Facultades de Ciencias de la Actividad Física y del Deporte (2004) sobre la situación laboral de estos licenciados en España determina que el $46 \%$ trabajan en la docencia de la asignatura de Educación Física, entre el 15\% y el 20\% están en la dirección deportiva y el entrenamiento deportivo, el $10 \%$ en la actividad física y salud y el $6 \%$ en la recreación deportiva. Asimismo, obtiene que el 6,1\% son autónomos, el 5\% no tiene contrato, el 38\% están pluriempleados, el $83 \%$ han estado en varios puestos de trabajo y la contratación temporal es del 50,6\%. Respecto a la jornada laboral, el 56,7\% trabajan entre 20 y 40 horas semanales, el 24,4\% menos de 20 horas y el 11,6\% más de 40 horas. 
Por todo ello, y considerando la importancia y relevancia de la situación profesional de los Licenciados en Ciencias de la Actividad Física y del Deporte, esta investigación analiza el perfil ocupacional y laboral de los licenciados en Ciencias de la Actividad Física y del Deporte. Los objetivos específicos de esta investigación con respecto a los licenciados en Ciencias de la Actividad Física y del Deporte que trabajan en la Comunidad Valenciana son:

- Describir las funciones laborales de actividad física y deporte realizadas;

- Establecer las características laborales y la tipología de las entidades en la que trabajan;

- Interaccionar las funciones laborales con las características laborales y las entidades en las que trabajan.

\section{METODOLOGÍA}

La metodología que se ha seguido en esta investigación ha sido cuantitativa de corte descriptivo. En el desarrollo de esta metodología, los procedimientos que se han seguido son los propios de una encuesta (ALVIRA, 2004). La población objeto de estudio la constituyeron las personas que trabajan desarrollando funciones laborales de actividad física y deporte en la Comunidad Valenciana.

El tamaño de la muestra fue de 600 personas, y trabajando con un intervalo de confianza del 95,5\%, y suponiendo en la varianza poblacional el caso más desfavorable de $\mathrm{p}$ igual a $50 \%$, luego $\mathrm{q}=50 \%$, el margen de error permitido de muestreo es de $\pm 4 \%$. El tipo de muestreo fue probabilístico de tipo polietápico, estratificado en primera fase, por conglomerados (FINK, 1995; CEA, 1998). La afijación de la muestra fue proporcional a la distribución de las personas según tamaño demográfico de los municipios de las provincias de Alicante, Castellón y Valencia y su situación geográfica. Además, se realizaron diversos submuestreos teniendo como objetivo dispersar la muestra para que influyera positivamente en la precisión de las estimaciones (RODRÍGUEZ, 2002).

Siguiendo a Miquel et al. (1996) y García Ferrando (2002), una vez establecidos los objetivos, se elaboró y validó el instrumento utilizado para obtener la información, que fue una entrevista de tipo oral, individual y estandarizada por medio de cuestionario compuesta de preguntas cerradas y categorizadas (SIERRA, 2001; BRYMAN, 2004). Con respecto a la validez de contenido fue validado por doce especialistas externos al equipo de investigación. Asimismo, se realizó una prueba piloto o pretest, siguiendo lo establecido por Miquel et al. (1996) y García Ferrando (2002), en donde el instrumento fue 
administrado a cincuenta personas perteneciente al universo estudiado. Todos los sujetos entendieron las cuestiones y alternativas de respuesta, y no hubo valores perdidos. Además, la entrevista estandarizada por medio de cuestionario ya había sido validada y utilizada en anteriores estudios sobre personas que trabajan en funciones de actividad física y deporte (CAMPOS IZQUIERDO, 2001, 2004). Después se preparó el trabajo de campo y se procedió a la recogida de datos, donde las 600 entrevistas, únicamente fueron realizadas por un único entrevistador, para tener una mayor fiabilidad y validez en el estudio, y éstas se realizaron de forma personal e individual cara a cara a cada uno de los individuos seleccionados de la muestra en las instalaciones deportivas (FINK, 1995; BRYMAN, 2004). Posteriormente, se realizó el procesamiento y análisis de la información obtenida mediante el paquete informático de programas SPSS para Windows versión 15.0.

\section{RESULTADOS}

Para contextualizar el análisis de los resultados, hay que señalar que del total de las personas que trabajan en funciones de actividad física y deporte en la Comunidad Valenciana, el 15,9\% son Licenciados en Ciencias de la Actividad Física y del Deporte, mientras que el 43,5\% son personas que tienen algunas de las otras titulaciones oficiales de la actividad física y deporte (véase Tabla 1) y el 40,6\% de personas no poseen ninguna de las diferentes titulaciones de actividad física y deporte.

En el análisis del porcentaje de realización de cada una de las funciones laborales realizadas por los Licenciados en Ciencias de la Actividad Física y del Deporte en la actualidad, se obtiene que el primer grupo de funciones más desarrolladas son: la docencia de la asignatura de Educación Física en el sistema educativo (31,5\% del total de las funciones realizadas), seguido por la dirección y organización de actividad física y deporte (en el que se incluye la inspección y asesoramiento) con el 18,5\%. El segundo grupo de funciones más desarrolladas son: el entrenamiento de equipos o individuos para la competición y el cuidado y mantenimiento de la forma física para grupos con porcentajes entre el $11 \%$ y $12 \%$. Posteriormente, se encuentran las funciones de entrenamiento personalizado, readaptación o reeducación física mediante ejercicio físico y la docencia de actividad física y deportes con porcentajes entre el 5\% y 7\%. Finalmente, se encuentran las actividades físico-deportivas fuera de horario lectivo en los centros educativos, la animación deportiva y la docencia de contenidos de actividad física y deporte en cursos de formación con porcentajes entre el $2 \%$ y $4 \%$ (Tabla 2). 
Tabla 2 - Porcentajes de las Funciones de Actividad Física y Deporte Realizadas en la Actualidad

Funciones laborales de actividad física y deporte

Docencia de la asignatura de Educación Física en el sistema educativo

Dirección y organización de actividad física y deporte

Entrenamiento de equipos o individuos para la competición

Cuidado y mantenimiento de la forma física en grupos

Docencia de actividad física y deportes

Entrenamiento personalizado

Readaptación o reeducación física mediante ejercicio físico

Actividades físico-deportivas fuera de horario lectivo en centros educativos

Animación deportiva

Docencia de contenidos de actividad física y deporte en cursos de formación
Porcentaje

$31,5 \%$

$18,5 \%$

$11,9 \%$

$11 \%$

$7 \%$

$\mathbf{5 , 5 \%}$

$5,5 \%$

$3,8 \%$

$3,2 \%$

$2,1 \%$

Al estudiar la realización de las funciones laborales de actividad física y deporte según la cantidad de personas que trabajan en cada una de las mismas, en la actualidad son más del 55\% los licenciados que imparten la docencia de la asignatura de Educación Física, y más del 30\% dirigen y organizan actividad física y deporte. Asimismo, más del $20 \%$ de estos licenciados, realizan el entrenamiento de equipos o individuos para la competición o el cuidado y mantenimiento de la forma física.

Al analizar la realización de una o varias funciones, se obtiene que en la actualidad hay una elevada polifuncionalidad por parte de los licenciados en Ciencias de la Actividad Física y del Deporte $(52,7 \%)$ donde más del $20 \%$ de los mismos realizan tres o más tipos de funciones diferentes, mientras que los que realizan una única función representan el 47,3\%. Además, la inmensa mayoría de los licenciados (96,7\%) en el pasado han realizado una o varias funciones laborales de actividad física y deporte diferentes a las que realizan actualmente (Tabla 3).

Tabla 3 - Porcentajes de Polifuncionalidad

\begin{tabular}{lc}
\hline Polifuncionalidad & Porcentaje \\
\hline Única Función & $\mathbf{5 2 , 7 \%}$ \\
\hline Varias funciones & $\mathbf{4 7 , 3 \%}$ \\
\hline
\end{tabular}

De los licenciados que en la actualidad realizan varias funciones de actividad física y deporte, las funciones que más desarrollan conjuntamente son la docencia de la asignatura de Educación Física con el entrenamiento de equipos o individuos para la competición y por otro lado la dirección y organización con el cuidado y mantenimiento de la forma física para grupos. 
$\mathrm{Al}$ analizar la preferencia de funciones laborales de actividad física y deporte, hay que resaltar que el $69,2 \%$ de los licenciados no desean trabajar en otras funciones de actividad física y deporte diferentes a las que realizan en la actualidad mientras que el $22 \%$ sí que lo desean y el 8,8\% tienen dudas. Dentro de las funciones deseadas diferentes a las que desarrollan en la actualidad, las principales son la docencia de la asignatura de Educación Física y la de dirección y organización de actividad física y deporte con porcentajes alrededor del 35\% cada una, seguido, con el 10\%, cada una, por el entrenamiento de equipos o individuos para la competición y la readaptación o reeducación física mediante ejercicios físicos.

Respecto a las relaciones laborales de los licenciados en Ciencias de la Actividad Física y del Deporte, el $15,2 \%$ de las mismas representan situaciones en las que no hay ningún tipo de contratación, mientras que el 79,2\% de las relaciones laborales son de algunos de los tipos de contratación posibles según la legislación vigente y el 5,6\% de las relaciones laborales son del tipo denominado "autónomo" (Tabla 4).

Tabla 4 - Porcentajes de las Diferentes Tipologías de Relaciones Laborales

\section{Relaciones laborales}

Sin contrato

Contrato

Autónomo

\section{Porcentaje}

$15,2 \%$

$79,2 \%$

$5,6 \%$

Asimismo, los que trabajan en la actualidad con más de una relación laboral representan el 31,9\% y con única relación laboral el 68,1\%.

Al hacer referencia las relaciones laborales en cada una de las funciones realizadas por los licenciados, se observa que la docencia de la asignatura de Educación Física, la dirección y organización de actividad física y deporte y el entrenamiento personal son los que mayor porcentajes de contratación presentan (superiores al 75\%). Por el contrario, el entrenamiento de equipos o individuos para la competición, las actividades físico-deportivas fuera del horario lectivo, la animación deportiva y la docencia de contenidos de actividad física y deporte son las funciones que mayor porcentaje tienen de no contratación (superior al 30\%). Asimismo, el entrenamiento personal y la readaptación mediante ejercicio físico son los que mayor porcentaje representan de autónomos (mas del 20\%) (Tabla 5). 
Tabla 5 - Porcentajes de las Relaciones Laborales en las Funciones de Actividad Física y Deporte

\begin{tabular}{|c|c|c|c|}
\hline Funciones laborales & Contrato & No contrato & Autónomo \\
\hline Docencia de la asignatura de Educación Física & $95,9 \%$ & $2,8 \%$ & $1,3 \%$ \\
\hline Dirección y organización de actividad física y deporte & $76,6 \%$ & $8,4 \%$ & $15 \%$ \\
\hline Entrenamiento de equipos o individuos para la competición & $59 \%$ & $38,7 \%$ & $2,3 \%$ \\
\hline Cuidado y mantenimiento de la forma física en grupos & $68,9 \%$ & $12,4 \%$ & $18,7 \%$ \\
\hline Docencia de actividad física y deportes & $68,7 \%$ & $11,8 \%$ & 19,5 \\
\hline Entrenamiento personalizado & $76,9 \%$ & $0 \%$ & $23,1 \%$ \\
\hline Readaptación o reeducación física mediante ejercicio físico & $65,3 \%$ & $13,4 \%$ & $21,3 \%$ \\
\hline Actividades físico-deportivas fuera de horario lectivo & $60 \%$ & $30 \%$ & $10 \%$ \\
\hline Animación deportiva & $60 \%$ & $30 \%$ & $10 \%$ \\
\hline Docencia de contenidos de actividad física y deporte en cursos & $66,6 \%$ & $33,4 \%$ & $0 \%$ \\
\hline
\end{tabular}

Entre los licenciados que están contratados, la contratación indefinida representa el 56\% de las contrataciones y la contratación temporal el $44 \%$. La mayoría de los contratos que se realizan son de dedicación a tiempo completo (74\% de los mismos), mientras a tiempo parcial son el 26\% (Tabla 6).

Tabla 6 - Porcentajes de Tipología de Contratación

\begin{tabular}{lc}
\hline \multicolumn{1}{|c|}{ Contratación } & Porcentaje \\
\hline Contratación temporal & $\mathbf{4 4 \%}$ \\
Contratación indefinida & $\mathbf{5 6 \%}$ \\
\hline
\end{tabular}

En cuanto a la tipología de contratación en cada una de las funciones realizadas por los licenciados, se observa que en la docencia de la asignatura de Educación Física y en la dirección y organización de actividad física y deporte el porcentaje de contratación indefinido es mayoritario. Por el contrario, en las restantes funciones laborales de actividad física y deporte la contratación temporal es mayoritaria (Tabla 7).

Tabla 7 - Porcentajes de Tipología de Contratación en las Funciones de Actividad Física y Deporte

\begin{tabular}{lcc}
\hline \multicolumn{1}{c}{ Funciones laborales } & Indefinido & Temporal \\
\hline Docencia de la asignatura de Educación Física en el sistema educativo & $\mathbf{6 2 , 2 \%}$ & $37,8 \%$ \\
Tareas de dirección y organización de actividad física y deporte & $\mathbf{6 2 , 2 \%}$ & $37,8 \%$ \\
Entrenamiento de equipos o individuos para la competición & $40,4 \%$ & $\mathbf{5 9 , 6 \%}$ \\
Cuidado y mantenimiento de la forma física en grupos & $43,5 \%$ & $\mathbf{5 6 , 5 \%}$ \\
Docencia de actividad física y deportes & $44,6 \%$ & $\mathbf{5 5 , 4 \%}$ \\
Entrenamiento personalizado & $45 \%$ & $\mathbf{5 5 \%}$ \\
Readaptación o reeducación física mediante ejercicio físico & $47,3 \%$ & $\mathbf{5 2 , 7 \%}$ \\
Actividades físico-deportivas fuera de horario lectivo en centros escolares & $40,9 \%$ & $\mathbf{5 9 , 1 \%}$ \\
Animación deportiva & $45,1 \%$ & $\mathbf{5 4 , 1 \%}$ \\
Docencia de contenidos de actividad física y deporte en cursos & $48 \%$ & $\mathbf{5 2 \%}$ \\
\hline
\end{tabular}

Conexões: revista da Faculdade de Educação Física da UNICAMP, Campinas, v. 8, n. 2, p. 01-18, maio/ago. 2010. 
La jornada laboral está muy diversificada, siendo la jornada entre 21 y 40 horas la mayoritaria con el $63 \%$ de las personas, le sigue las que trabajan más de 40 horas (29\%), después menos de 21 horas trabajan el $8 \%$ de las personas.

La duración de las relaciones laborales con las entidades para las que trabajan en la actualidad predomina por encima de 3 años (58\%), donde sólamente llevan más de 10 años trabajando el 29,4\% de las relaciones laborales.

Las entidades para las que los Licenciados en Ciencias de la Actividad Física y del Deporte trabajan son diversas. Dentro de estas entidades, las mayoritarias son las entidades públicas, ya que representan el $50,4 \%$. Después están las empresas (entidades con ánimo de lucro) con el 32\% del total de las entidades empleadoras y le siguen las asociaciones o federaciones (entidades sin ánimo de lucro) que representa el $17,6 \%$ (Tabla 8).

Tabla 8 - Porcentajes de Entidades

\begin{tabular}{lc}
\multicolumn{1}{c}{ Entidades } & Porcentaje \\
\hline Entidad Pública & $\mathbf{5 0 , 4 \%}$ \\
Empresa (entidades con animo de lucro) & $\mathbf{3 2 \%}$ \\
Asociaciones o federaciones (entidades sin ánimo de lucro) & $\mathbf{1 7 , 6 \%}$ \\
\hline
\end{tabular}

Al hacer referencia a las entidades en cada una de las funciones realizadas por estos licenciados se observa que en la docencia de la asignatura de Educación Física, la dirección y organización de actividad física y deporte son mayoría los licenciados que trabajan en entidades públicas. Sin embargo, en el entrenamiento personal, las actividades físico-deportivas fuera del horario lectivo y la readaptación

mediante ejercicio físico la mayoría de los licenciados trabajan en empresas. Asimismo, en el entrenamiento de equipos o individuos para la competición, mayoría de los licenciados trabajan en asociaciones (Tabla 9). 
Tabla 9 - Porcentajes de Ttipología de las Entidades en las Funciones de Actividad Física y Deporte

\begin{tabular}{|c|c|c|c|}
\hline Funciones laborales & Entidad & Empresa & Asociaciones \\
\hline & & & \\
\hline Docencia de la asignatura de Educación Física & $67,6 \%$ & $32,4 \%$ & $0 \%$ \\
\hline Dirección y organización de actividad física y deporte & $\mathbf{5 5 , 8 \%}$ & $28,9 \%$ & $15,3 \%$ \\
\hline Entrenamiento de equipos o individuos para la competición & $23,1 \%$ & $10,8 \%$ & $66,4 \%$ \\
\hline Cuidado y mantenimiento de la forma física en grupos & $40,7 \%$ & $44,1 \%$ & $15,2 \%$ \\
\hline Docencia de actividad física y deportes & $47,8 \%$ & $35,9 \%$ & $16,3 \%$ \\
\hline Entrenamiento personalizado & $36,8 \%$ & $55,3 \%$ & $7,9 \%$ \\
\hline Readaptación o reeducación física mediante ejercicio físico & $37,5 \%$ & $54,2 \%$ & $8,3 \%$ \\
\hline Actividades físico-deportivas fuera de horario lectivo & $31,1 \%$ & $50,2 \%$ & $18,7 \%$ \\
\hline Animación deportiva & 36,2 & $40,6 \%$ & $23,2 \%$ \\
\hline Docencia de contenidos de actividad física y deporte en c & $36,4 \%$ & $39,4 \%$ & $24,2 \%$ \\
\hline
\end{tabular}

Además, de los Licenciados en Ciencias de la Actividad Física y del Deporte que anteriormente han trabajado en otras entidades diferentes a la actual, más del $90 \%$ lo han hecho en varias entidades. De este porcentaje entre dos y cuatro entidades diferentes han trabajado el 55,6\% y los que han trabajado entre cinco y nueve entidades representan el $33 \%$.

El 71,4\% de los Licenciados no están colegiados en el Colegio Profesional de la Comunidad Autónoma Valenciana, mientras que los que están colegiados son el 28,6\% de los Licenciados en Ciencias de la Actividad Física y del Deporte.

\section{DISCUSIÓN}

Los Licenciados en Ciencias de la Actividad Física y del Deporte realizan todos los tipos de funciones de actividad física y deporte, y se observa la tendencia en las funciones laborales de crecimiento y diversificación de las mismas (CAMERINO; MIRANDA; PIGEASSOÚ, 1995; MARTÍNEZ DEL CASTILLO; PUIG, 2002; CAMPOS IZQUIERDO, 2008). La función más realizada, de forma destacada, es la docencia de la asignatura de Educación Física, al igual que exponen Rivadeneyra (1998) y Puig; Viñas (2006), sin embargo ha descendido respecto a lo obtenido por Martínez del Castillo (1991). Después, le sigue la dirección y organización de actividad física y deporte (18,5\%), cuyo porcentaje es superior al 10,6\% que obtiene Martínez del Castillo (1991) e indica su aumento como empleo, tal como exponen Quain y Parks (1986), Consejo Superior de Deportes (2000) y Pérez; Stucchi (2007). Hay que remarcar que ambas funciones laborales son las mas deseadas y que según se avanza en edad se tiende a ser profesores de Educación Física o directores de actividad física y deporte, ya que son las que mejores condiciones labores y profesionales presentan (PUIG; VIÑAS, 2006; CAMPOS IZQUIERDO; PABLOS; 
MESTRE, 2006; MADELLA, 2002). Seguidamente se encuentran, con porcentajes entre el $11 \%$ y $12 \%$, el entrenamiento de equipos o individuos para la competición y el cuidado y mantenimiento de la forma física para grupos, las cuales disminuyen respecto al porcentaje obtenido por Martínez del Castillo (1991). También, se observan dos tendencias, la primera, un aumento respecto a lo obtenido por Martínez del Castillo (1991) en las funciones de entrenamiento personalizado y la readaptación o reeducación física mediante ejercicio físico; y la segunda, el descenso por parte de la docencia de actividad física y deportes, las actividades físico-deportivas extraescolares y la animación deportiva.

Igualmente, se observa una gran polifuncionalidad de los licenciados, tanto en la actualidad como a lo largo de su vida laboral, en la que todas las funciones se desarrollan conjuntamente con la mayoría de las otras funciones, al igual que reflejan los estudios de Camy, Chantelat y Le Roux (1999) y González (2008). Ésta puede ser un factor interesante a tener en cuenta en la formación de estos titulados.

En relación con los aspectos laborales, se determina que más del 15\% de las relaciones laborales de las personas que trabajan en funciones de actividad física y deporte representa situaciones en las que no hay ningún tipo de contratación. Además, estos porcentajes son superiores a lo obtenido por Martínez del Castillo (1991), por lo que se puede deducir que la no contratación ha aumentado en estas últimas décadas. También, hay que resaltar que la no contratación se produce en todos los tipos de organizaciones de actividad física y deporte y en todos los tipos de funciones de actividad física y deporte, aunque la no contratación es minoritaria en la docencia de la asignatura de Educación Física, en la dirección y organización de actividad física y deporte y en el entrenamiento personal.

Un aspecto que cabe resaltar es que el porcentaje de autónomos ha subido significativamente respecto al obtenido por Martínez del Castillo (1991), e incluso en las funciones de entrenamiento personal y readaptación física mediante ejercicio físico el porcentaje de autónomos está por encima del $20 \%$ lo que corrobora la opinión de Le Roux y Frosberg (2000) y Primault (2006) sobre el aumento del autoempleo en estas funciones en las últimas décadas en España.

Asimismo, el 44\% de los contratos son temporales, porcentaje muy superior al obtenido por Martínez del Castillo (1991) y al de la población a nivel general de España (Instituto Nacional de Estadística, 2008). Todo ello supone una elevada temporalidad, que además ha aumentado en las últimas décadas. 
La dedicación horaria está muy diversificada. Las personas que trabajan más de 40 horas representan el 29\%, porcentaje superior al 15,5\% obtenido por Martínez del Castillo (1991) y Campos Izquierdo, Pablos; Mestre (2006). Esto puede indicar la tendencia, en estos titulados, del aumento de las jornadas laborales mayores de 40 horas.

Las entidades para las que los Licenciados trabajan son diversas. Mayoritariamente trabajan en entidades públicas, al igual que ocurre en los estudios de Augustin (2003) y Almeida (2007), ya que éstas representan el 50,4\%, aunque se produce un gran retroceso respecto a lo obtenido por Martínez del Castillo (1991). Las empresas de actividad física y deporte en la actualidad son el 32\%, y se produce un considerable aumento de personas que trabajan en las mismas respecto a Martínez del Castillo (1991). Seguidamente, se encuentran las asociaciones o clubes deportivos, ya que estas son un factor interesante de empleo en la actividad física y deporte (HEINEMANN, 1998).

Respecto a la identificación y asociación con una organización corporativa y profesional propia para la potenciación de la profesionalización de estos titulados y su mercado laboral y consideración como profesión, según lo expuesto por Camy, Chantelat y Le Roux (1999), Almeida (2007) y Campos Izquierdo (2010) resulta ser escasa, ya que sólamente el 28,6\% de los Licenciados están colegiados en el Colegio Profesional de la Comunidad Valenciana.

\section{CONCLUSIONES}

En los Licenciados en Ciencias de la Actividad Física y del Deporte se observa la tendencia de crecimiento y diversificación de las funciones de actividad física y deporte, aunque las mayormente realizadas son: la docencia de la asignatura de Educación Física, que constituye la más destacada, aunque ha decrecido en las últimas décadas; la dirección y organización de actividad física y deporte, que han aumentado en las últimas décadas; el entrenamiento de equipos o individuos y el cuidado y mantenimiento de la forma física para grupos, las cuales han disminuido en estas últimas décadas. Hay que remarcar que según se avanza en edad se tiende a ser profesores de Educación Física o directores de actividad física y deporte, ya que son las más deseadas y las que mejores condiciones labores y profesionales presentan.

La plurifuncionalidad es mayoritaria en estos titulados, cuestión interesente para abordar en la formación de estos titulados. 
Las relaciones laborales sin contrato son significativas, y se produce en todas las funciones laborales y entidades, además, ésta ha aumentado en estas ultimas décadas. Asimismo, el porcentaje de autónomos está aumentando, lo que indica la tendencia de estos titulados al autoempleo y mucho más en el entrenamiento personal y la readaptación física mediante ejercicio físico. También es elevado y ha aumentado el porcentaje de personas con contrato temporal, movilidad laboral y las que trabajan más de 40 horas, lo que conforma cierta precariedad e inestabilidad laboral por parte de los Licenciados en Ciencias de la Actividad Física y del Deporte, aunque es inferior a la mayoría de los otros titulados.

La mayoría de estos Licenciados trabajan para diversas entidades, pero principalmente lo hacen para entidades públicas, aunque los que trabajan en empresas de actividad física y deporte han aumentado considerablemente en las últimas décadas. Asimismo, el porcentaje de asociados en el colegio profesional es muy escaso.

\section{REFERENCIAS}

ALMEIDA, C. $O$ emprego e as profissoes do desporto. In: OLIMPO, J.; CONSTANTINO, J. M. (Coord.). Em defesa do desporto. Coimbra: Almedina, 2007. p. 123-145.

ALVIRA, F. La encuesta: una perspectiva general metodológica. Madrid: C.I.S., 2004

AUGUSTIN, J. P. Le sport et ses metiers: nouvelles pratiques et enjeux d'une professionnalisation. Paris: La Découverte, 2003.

BRYMAN, A. Social research methods. New York: Oxford University Press, 2004.

CAMERINO, O.; MIRANDA, J.; PIGEASSOÚ, C. H. La actividad física y el ocio: análisis del mercado y planificación de la formación. Rev Esp Educ Fis Dep, n. 1, p. 34-38, 1995.

CAMPOS IZQUIERDO, A. La situación actual de los profesionales de la actividad física y el deporte en las instalaciones deportivas de la Comarca Burgalesa de la Ribera del Duero. Valencia: Universidad de Valencia, 2001.

- Los profesionales de la actividad física y deporte y la organización-regulación de su mercado laboral. Act Fis Cien Prof, n. 5, p. 14-19, 2004.

Situación profesional de las personas que trabajan en funciones de actividad física y deporte en la Comunidad Autónoma Valenciana (2005). Valencia: Universidad de Valencia, 2007a.

. Los profesionales de la actividad física y deporte como elemento de garantía y calidad de los servicios. Cult Cien Dep n. 7, p. 51-57, 2007 b. 
CAMPOS IZQUIERDO, A. La organización de los recursos humanos de la actividad física y deporte desde la perspectiva de sus funciones. Motricidad, n. 21, p. 142-167, 2008.

. Dirección de recursos humanos en las organizaciones de la actividad física y del deporte. Madrid: Síntesis, 2010.

.; PABLOS, C.; MESTRE J. A. Los titulados de la actividad física y del deporte: evolución histórica y perfiles profesionales. Sevilla: Wanceulen, 2006.

CAMY, J.; CHANTELAT, P.; LE ROUX, N. Sport et emploi en Europe. Paris: Comisión Europeenne, 1999.

CASTEllani FILHO, L. Política educacional e Educação Física. Campinas: Autores Associados, 2002.

CEA, M. A. Metodología cuantitativa: estrategias y técnicas de investigación social. Madrid: Síntesis, 1998.

CONFERENCIA de decanos de las facultades de ciencias de la actividad física y del deporte. Propuesta de grado en Ciencias de la Actividad Física y el Deporte. Cáceres: Universidad de Extremadura, 2004.

DANTAS, R. J. Do senso comum à ciencia: paro o profissional de educaçao física, uma transiçao difícil de ser completamente comprendida. Conexões, Campinas, v. 6, n. 2, p. 82-89, 2008.

FINK, A. How to sample in surveys. Thousand Oaks: Sage, 1995.

GARCÍA FERRANDO, M. La encuesta. In: ALVIRA, F.; GARCÍA FERRANDO, M.; IBÁÑEZ, J. (Org.). El análisis de la realidad social: métodos y técnicas de investigación. Madrid: Alianza, 2002. p. 167-202.

GONZÁLEZ, M. El deporte escolar en la comunidad autónoma de Madrid: intervención didáctica y recursos humanos en las actividades físico-deportivas extraescolares en los centros educativos. Valencia: Universidad de Valencia, 2008.

HEINEMANN, K. Introducción a la economía del deporte. Barcelona: Paidotribo, 1998.

JIMÉNEZ, I. El ejercicio profesional de las titulaciones del deporte. Barcelona: Bosch, 2001.

LE ROUX, N.; FROSBERG, K. ENSSHE and the trends in sport and employment in Europe. Bull Int Counc Sport Sci Phys Educ, n. 30, p. 22-24, 2000.

MADELLA, A. Le professioni dello sport. SDS: Rivista di Cultura Sportiva, vol. 21, n. 5, p. 2-9, 2002.

. Methods for analysing sports employment in Europe. Manag Leis, v. 8, n. 2, p. 56-69, 2003.

MADRID. Instituto Nacional de Estadística. Encuesta de Población activa. Madrid: INE., 2008. 
MADRID. Ministerio de Educação e Cultura. Consejo Superior de Deportes. El deporte español ante el siglo XXI. Madrid: MEC: CSD, 2000.

. Ministerio de Educação e Cultura. Consejo Superior de Deportes. Borrador de anteproyecto de Ley sobre ordenación del ejercicio de determinadas profesiones del deporte. Madrid: MEC: CSD, 2009. MARTÍNEZ DEL CASTILLO, J. La estructura ocupacional del deporte en España. Encuesta realizada sobre los sectores de Entrenamiento, Docencia, Animación y Dirección. Madrid: CSD, 1991.

.; PUIG, N. Mercado de trabajo en el deporte. In: GARCÍA FERRANDO, M.; LAGARTERA, F.; PUIG, N. (Org.). Sociología del deporte. Madrid: Alianza, 2002. p. 283-316.

MCMURTIE, R. Sports employment trends in Australia. Sport Educ, v. 9, n. 2, p. 23-24, 1997.

MIQUEL, S. et al. Investigación de mercados. Madrid: McGraw-Hill, 2000.

PEREZ, T.; STUCCHI, S. Relações preliminares entre a gestão esportiva e o profissional de Educação Física. Conexões, Campinas, v. 5, n. 2, p. 113-128, 2007.

PETRY, K.; FROBERG, K.; MADELLA, A. Higher education in sport in Europe: from labour market demand to training supply. Aaachen: Meyer \& Meyer, 2008.

PRIMAULT, D. Employment in sport. In: ANFREFF, W.; SZYMANSKI, S. (Org.). Handbook on the economics of sport. Cheltenham: Edward Elgar, 2006. p. 153-167.

PUIG, N.; VIÑAS, J. Estructura ocupacional de licenciados y licenciadas en el INEF-Cataluña, Barcelona (1980-2005). Barcelona: INEFC-Cataluña, 2006.

QUAIN, R.; PARKS, J. Sport management survey: employment perspectives. J Phys Educ Recreat Dance, v. 57, n. 4, p. 18-21, 1986.

RIVADENEYRA, M. L. Perspectivas laborales y formación inicial de los Licenciados en Ciencias de la Actividad Física y Deporte de la Universidad de Granada. Granada: Universidad de Granada, 1998.

RODRÍGUEZ, J. La muestra: teoría y aplicación. In: ALVIRA, F.; GARCÍA FERRANDO, M.; IBÁÑEZ, J. (Org.). El análisis de la realidad social: métodos y técnicas de investigación. Madrid: Alianzal, 2002. p. 445-484.

SIERRA, R. Técnicas de investigación social: teoría y ejercicios. Madrid: Paraninfo, 2001.

TAKS, M. et al. Tracking the careers of graduates in sport and sport-related programmes: a European perspective. Manag Leis, v. 8, n. 2, p. 97-103, 2003.

TOJAL, J. B. A. G. Da Educaçao Física à motricidades humana: a preparação do profissional. Lisboa: Instituto Piaget, 2004. 
VERENGUER, R. C. G. Mercado de trabalho em Educacão Física: reestructuraçao produtiva, relações de trabalho e intervenção profissional. Rev Mackenzie Educ Fis Esporte, v. 4, n. 4, p. 34-54, 2005.

\section{ANTONIO CAMPOS IZQUIERDO}

Universidad Politécnica de Madrid, España

MARÍA DOLORES GONZÁLEZ RIVERA

Universidad de Alcalá, Madrid, España

\section{Referência do artigo}

\section{ABNT}

CAMPOS IZQUIERDO, A.; GONZALEZ RIVERA, M. D. El perfil laboral de los licenciados en ciencias de la actividad física y del deporte en la comunidad valenciana, españa. Conexões, v. 8, n. 2, p. 1-18, 2010.

APA

Izquierdo, A. C., \& Rivera, M. D. G. (2010). El perfil laboral de los licenciados en ciencias de la actividad física y del deporte en la comunidad valenciana. Conexões, 8(2), 1-18.

\section{VANCOUVER}

Izquierdo, AC, Rivera, MDG, El perfil laboral de los licenciados en ciencias de la actividad física y del deporte en la comunidad valenciana. Conexões, 2010; 8 (2): 1-18.

Recebido em: nov./2009

Aceito para publicação em: mar./2010 